Im „Journal Club“ werden Originalarbeiten aus der internationalen Fachliteratur referiert und kommentiert.

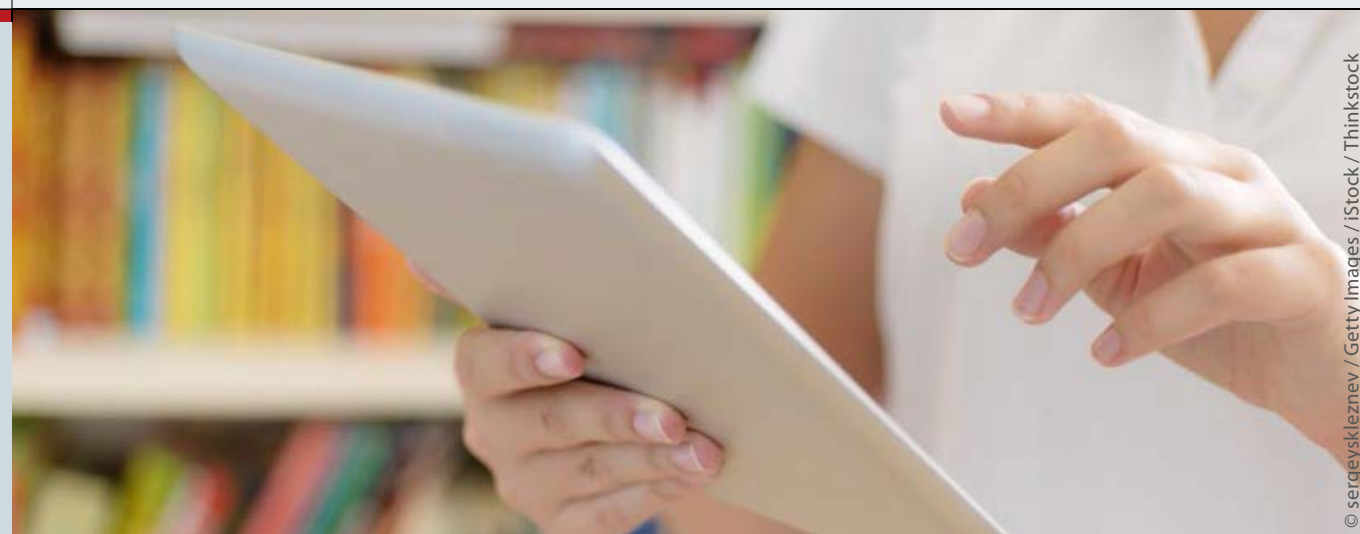

\section{Antibiotikasäfte: Jede zweite Dosierung ist falsch}

\section{Eine Suspension korrekt herzustellen scheint gar nicht so einfach zu sein, wie eine Studie mit 100 Erwachse- nen jetzt zeigte.}

$\mathrm{E}^{\mathrm{h}}$ ltern machen jede Menge Fehler, - wenn es darum geht, ihrem Sprössling eine flüssige Medizin zu verabreichen. 100 Erwachsene, die für die Versorgung eines erkrankten Kindes zuständig waren, nahmen an der prospektiven Beobachtungsstudie teil. Sie erhielten entweder ein Rezept für Amoxicillin mit Dosierlöffel oder für Josamycin mit Dosierpipette. Nachdem sie von einem Apotheker über die Anwendung des Antibiotikums informiert worden waren, stellten sie die Suspension her und dosierten die verschriebene Menge. Danach wurden sie per Fragebogen $\mathrm{zu}$ ihrem allgemeinen Wissen $\mathrm{zu}$ oralen Flüssigmedikationen befragt. Die Fehler, die die Eltern beim Ansetzen der Antibiotikasäfte machten, brachten etwa bei jeder zweiten Zubereitung die Gefahr der Über- oder Unterdosierung mit sich. So wurde beispielsweise das Wasser nicht bis zur richtigen Markierung aufgefüllt, nicht genügend geschüttelt, so dass die Suspension klumpig blieb, und nicht ausreichend lang bis zum Absetzen des Schaumes gewartet. 56\% der Eltern schafften es nicht, mit dem Dosierlöffel die richtige Menge abzumessen. Weniger Probleme bereitete offenbar die Dosierspritze, hier lag die Fehlerquote bei $10 \%$.

\title{
Tiefe Wundinfektionen: Rauchen verdoppelt das Risiko
}

Dass Rauchen einen Einfluss auf die Wundheilung ausübt, konnte eine Analyse von 78.191 Patienten mit einer Hüft- oder Knie-TEP jetzt erneut belegen.

R auchen mindert den Blutfluss im Gewebe, löst eine Hypoxie aus und senkt die Kollagensynthese. Nach ermutigenden Hinweisen aus einigen kleineren Studien, dass es sich mit Blick auf die postoperative Komplikationen lohnt, Raucher vor einem Hüft- oder Kniegelenkersatz dem Tabak zu entwöhnen, haben Forscher der University of Iowa jetzt zu diesem Thema die Krankheitsverläufe von 78.191 Patienten mit Hüft- oder Knie-TEP analysiert.

Die bewerteten Infos sind in der Datenbank des American College of Surgeons National Surgical Quality Improvement Program (ACS NSQIP) niedergelegt. $82 \%$ der Patienten waren Nichtraucher, $8 \%$ ehemalige und $10 \%$ aktive Raucher. Die univariate Analyse ergab für die ersten 30 postoperativen Tage eine höhere Rate an Wundkomplikationen unter den Aktivrauchern; sie lag bei $1,8 \%$ verglichen mit 1,3 respektive $1,1 \%$ bei den Ex- und Nichtrauchern.

Um Differenzen zwischen den Gruppen auszugleichen, wurde eine multivariate Analyse durchgeführt. Sie ergab ein um $47 \%$ erhöhtes Risiko für tiefe Wundinfektionen bei Rauchern gegenüber Nichtrauchern. In puncto Gesamtkomplikationen lagen Raucher und Exraucher etwa gleichauf, ihr Risiko gegenüber den Nichtrauchern lag rund 20\% höher. Keine Unterschiede waren bei der Mortalität festzustellen.

J Bone Joint Surg Am 2015; 97 (13): 1049-58 